\title{
Three-dimensional flows around low-aspect-ratio flat-plate wings at low Reynolds numbers
}

\author{
KUNIHIKO TAIRA $†$ AND TIM COLONIUS \\ Division of Engineering and Applied Science, California Institute of Technology, \\ Pasadena, CA 91125, USA
}

(Received 18 March 2007 and in revised form 4 November 2008)

Three-dimensional flows over impulsively translated low-aspect-ratio flat plates are investigated for Reynolds numbers of 300 and 500, with a focus on the unsteady vortex dynamics at post-stall angles of attack. Numerical simulations, validated by an oil tow-tank experiment, are performed to study the influence of aspect ratio, angle of attack and planform geometry on the wake vortices and the resulting forces on the plate. Immediately following the impulsive start, the separated flows create wake vortices that share the same topology for all aspect ratios. At large time, the tip vortices significantly influence the vortex dynamics and the corresponding forces on the wings. Depending on the aspect ratio, angle of attack and Reynolds number, the flow at large time reaches a stable steady state, a periodic cycle or aperiodic shedding. For cases of high angles of attack, an asymmetric wake develops in the spanwise direction at large time. The present results are compared to higher Reynolds number flows. Some non-rectangular planforms are also considered to examine the difference in the wakes and forces. After the impulsive start, the time at which maximum lift occurs is fairly constant for a wide range of flow conditions during the initial transient. Due to the influence of the tip vortices, the three-dimensional dynamics of the wake vortices are found to be quite different from the two-dimensional von Kármán vortex street in terms of stability and shedding frequency.

\section{Introduction}

The Reynolds number at which micro-air-vehicles operate have reached $\mathcal{O}\left(10^{4}\right)$ and will continue to decrease in the coming years (Pines \& Bohorquez 2006). Due to the operational and weight requirements, these aircraft have unique designs with lowaspect-ratio wings, when compared to those of the conventional aircraft. Moreover, these vehicles fly at low speed and often high angles of attack while experiencing large perturbations such as wind gusts. Yet there are only few studies of low-Reynoldsnumber aerodynamics around low-aspect-ratio wings under translation.

As micro-air-vehicles become smaller in size, they share some characteristics with flying and swimming animals such as birds, insects and fishes. These animals have low-aspect-ratio wings or fins that are operating at Reynolds numbers of order $10^{2}$ to $10^{5}$, often at post-stall angles of attack. However, separated flows have been used to the advantage of the animals for enhanced performance. For example, it has been observed that hovering insects can achieve added lift from the unsteady

$\dagger$ Present address for correspondence: Department of Mechanical and Aerospace Engineering, Princeton University, Princeton, NJ 08544, USA. Email: ktaira@princeton.edu. 
separated flows around the flapping wings. The leading-edge vortices are found to be stably attached throughout each stroke and provide additional spanwise circulation resulting in enhanced lift. Such forces applied by the unsteady vortex formation in twodimensional flows were investigated both experimentally by Dickinson \& Götz (1993) and numerically by Wang $(2000 a, b, 2004)$ and Bos et al. (2008). Furthermore, the full three-dimensional studies drawing attention to spanwise flows and tip effects around the flapping wings were undertaken experimentally by Ellington et al. (1996), Birch \& Dickinson (2001), Usherwood \& Ellington (2002), Birch, Dickson \& Dickinson (2004) and Poelma, Dickson \& Dickinson (2006). Analogous numerical studies were performed by Liu \& Kawachi (1998) and Sun (2005).

Similar investigations have been carried out to analyse the efficient locomotion of swimming animals. In particular, von Ellenrieder, Parker \& Soria (2003), Buchholz \& Smits (2006) and Parker, von Ellenrieder \& Soria (2007) have visualized the wake vortices behind flapping low-aspect-ratio hydrofoils to understand the qualitative dynamics. Numerical simulations of the three-dimensional flows over flapping foils were also performed by Blondeaux et al. (2005) and Dong, Mittal \& Najjar (2006) at Reynolds numbers similar to the present study. Moreover, Drucker \& Lauder (1999) have experimentally visualized the flows around pectoral fins and Zhu et al. (2002) have performed simulations around an entire fish to identify the wake structures.

For purely translating low-aspect-ratio wings, Torres \& Mueller (2004) have experimentally measured the aerodynamic characteristics of low-aspect-ratio wings at Reynolds numbers around $10^{5}$. Aerodynamic performance (lift, drag, pitching moment, etc.) of various planforms was considered over angles of attack $(\alpha)$ of $0^{\circ}-$ $40^{\circ}$ and aspect ratios $(A R)$ of $0.5-2$ and was observed to be quite different from that of low-aspect-ratio wings in high-Reynolds-number flows. They concluded that the most important parameter that influences the aerodynamic characteristics is the aspect ratio. Transient studies have also been conducted by Freymuth, Finaish \& Bank (1987) by using smoke to visualize the start-up flows around low-aspectratio airfoils. A qualitative insight into the three-dimensional formation of wake vortices was presented. The experiments by Ringuette, Milano \& Gharib (2007) extensively studied the wake vortices behind low-aspect-ratio plates but only at $\alpha=90^{\circ}$.

On the numerical side, two-dimensional simulations around translating wings were performed by Hamdani \& Sun (2000). Also, studies by Mittal \& Tezduyar (1995) and Cosyn \& Vierendeels (2006) considered the three-dimensional flows around translating low-aspect-ratio planforms but focused mostly on those at low angles of attack. For wings at post-stall angles of attack, unsteady separated flows and vortex dynamics behind low-aspect-ratio wings in pure translation are still not well documented.

To extend the previous studies, we use numerical simulations to examine the aerodynamics of impulsively started low-aspect-ratio flat-plate wings under pure translation at Reynolds numbers of 300 and 500. These Reynolds numbers are high enough to induce separation and unsteadiness in the wake but low enough for the three-dimensional flow field to remain laminar. The regime also includes, for a range of angles of attack, the critical Reynolds numbers at which the flow first becomes unstable to small disturbances. In the following section, we present the numerical method and its validation. In $\S 3$, results from separated flows around the airfoils are presented. We call attention to the transient nature of the flow field and its influence on the aerodynamic forces. The stability of the wake at large time is also investigated. 
Dynamics of the wake vortices and the corresponding lift and drag are considered over a range of angles of attack and for various planform geometries.

\section{Simulation methodology}

\subsection{Numerical formulation}

Three-dimensional incompressible flow over a low-aspect-ratio flat-plate wing is simulated with an immersed boundary method (Peskin 2002; Mittal \& Iaccarino 2005; Taira \& Colonius 2007). With this method, a body of arbitrary geometry can be generated in a flow field by adding a boundary force to the momentum equation. The continuous analogue of the immersed boundary formulation can be represented by the following non-dimensional incompressible Navier-Stokes equations:

$$
\begin{aligned}
\frac{\partial \boldsymbol{u}}{\partial t}+\boldsymbol{u} \cdot \nabla \boldsymbol{u} & =-\nabla p+\frac{1}{R e} \nabla^{2} \boldsymbol{u}+\int_{s} \boldsymbol{f}(\boldsymbol{\xi}(s, t)) \delta(\boldsymbol{\xi}-\boldsymbol{x}) \mathrm{d} s \\
\nabla \cdot \boldsymbol{u} & =0 \\
\boldsymbol{u}(\boldsymbol{\xi}(s, t)) & =\int_{\boldsymbol{x}} \boldsymbol{u}(\boldsymbol{x}) \delta(\boldsymbol{x}-\boldsymbol{\xi}) \mathrm{d} \boldsymbol{x}=\boldsymbol{u}_{B}(\boldsymbol{\xi}(s, t)),
\end{aligned}
$$

where $\boldsymbol{u}, p$ and $\boldsymbol{f}$ are the appropriately non-dimensionalized velocity, pressure and surface force. The temporal variable is reported in terms of the non-dimensional convective time unit (i.e. $U_{\infty} t / c$ ). The spatial variables in the computational domain $\mathscr{D}$ and along the immersed boundary $\partial \mathscr{B}$ (in this case, surface of the plate) are denoted by $\boldsymbol{x}$ and $\boldsymbol{\xi}$, respectively, and are normalized by the mean chord of the plate. Reynolds number is defined as $R e \equiv U_{\infty} c / v$, where $U_{\infty}, c$ and $v$ denote the free-stream velocity, mean chord length of the plate and kinematic viscosity of the fluid, respectively. The velocity vector (non-dimensionalized by the free-stream velocity) is set to $\boldsymbol{u}_{B}$, the immersed boundary velocity, along $\partial \mathscr{B}$.

In later discussions, forces on the flat plate $\left(F_{x}, F_{y}, F_{z}\right)$ are described in terms of the non-dimensionalized lift, drag and side forces defined by $C_{L}=F_{y} /\left(\frac{1}{2} \rho U_{\infty}^{2} A\right)$, $C_{D}=F_{x} /\left(\frac{1}{2} \rho U_{\infty}^{2} A\right)$ and $C_{S}=F_{z} /\left(\frac{1}{2} \rho U_{\infty}^{2} A\right)$, respectively, where $\rho$ is the density of the fluid and $A$ is the area of the flat plate. In the case of two-dimensional flows, the force per unit span is normalized by the chord.

In the current work, we employ a particular immersed boundary method called the immersed boundary projection method developed by Taira \& Colonius (2007). This method enforces both the incompressibility and no-slip constraints through a single projection in a manner similar to the projection utilized to satisfy incompressibility in the traditional fractional-step methods. Equations (2.1)-(2.3) are spatially discretized through a second-order finite-volume formulation on a staggered grid. The convective and viscous terms are integrated in time with the second-order Adams-Bashforth and the Crank-Nicolson schemes, respectively. Further details on the method can be found in Taira \& Colonius (2007) and Taira (2008).

\subsection{Simulation setup}

Simulations are performed in a large rectangular box typically of size $[-4,6.1] \times[-5,5] \times[-5,5]$ in the streamwise $(x)$, vertical $(y)$ and spanwise $(z)$ directions. Typical grid size ranges from $125 \times 55 \times 80$ to $200 \times 88 \times 128$ with the smallest resolution of $\Delta x=0.025$ for the case of $A R=2$ and much larger sizes were used for simulations of flows around higher aspect ratio plates. Grid stretching is applied in all directions with finer resolution near the plate to capture the wake structure as illustrated in figure 1. Extensive studies have been performed in two and 


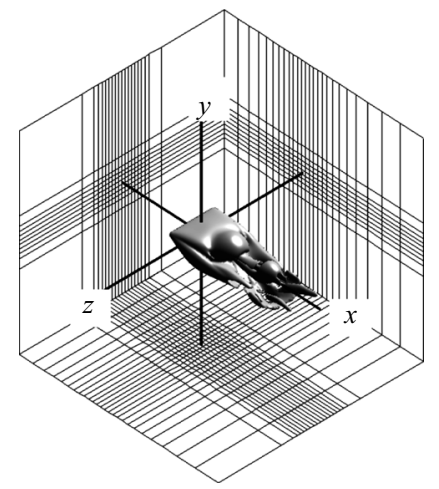

FIGURE 1. A typical computational domain showing the top-port side of the wake around a rectangular flat plate of $A R=2$. The spatial discretization of this computational domain is shown for every five cells for the $x$ - and $y$-directions and four cells for the $z$-direction.

three dimensions to ensure that the present choice of grid resolution and domain size does not influence the flow field in a significant manner (previously reported in Taira et al. 2007 and Taira 2008).

Boundary conditions along all sides of the computational boundary $\partial \mathscr{D}$ are set to uniform flow $\left(U_{\infty}, 0,0\right)$ except for the outlet boundary where a convective boundary condition $\left(\partial \mathbf{u} / \partial t+U_{\infty} \partial \mathbf{u} / \partial x=0\right)$ is specified. Inside the computational domain, a flat plate is positioned with its centre at the origin. This flat plate is instantaneously materialized at $t=0^{+}$in an initially uniform flow to model an impulsively started translating plate. Computations are advanced in time with a time step such that the Courant number based on the free-stream velocity obeys $U_{\infty} \Delta t / \Delta x \leqslant 0.5$. Both the initial transient and the large-time behaviour of the flow are considered.

\subsection{Validation}

We compare results from the three-dimensional simulations and measurements from an oil tow-tank experiment of flows over a rectangular plate of dimension $82 \mathrm{~mm} \times$ $164 \mathrm{~mm} \times 3 \mathrm{~mm}(A R=2)$. The experiment is performed in a tank $(2.4 \mathrm{~m} \times 1.2 \mathrm{~m} \times$ $1 \mathrm{~m})$ filled with a blend of Chevron Superla ${ }^{\circledR}$ white oil, Texaco Corporation. The rectangular flat plate is translated in the tank while being rigidly mounted to a six-axis force sensor at one-wing tip to limit lift due to backlash in the gearbox. This setup is attached to a translation sled equipped with a servomotor providing control of the translational velocity (Dickson \& Dickinson 2004). A constant translation velocity is maintained by the plate after a swift acceleration from rest. The temperature in the laboratory is regulated at $18 \pm 1^{\circ} \mathrm{C}$ such that there is less than $5 \%$ variation in the kinematic viscosity $(v=115 \mathrm{cSt})$. The variation in the translational velocity is much less than that of the viscosity, with the presence of the velocity control mechanism. Hence, the Reynolds number with estimated variation is $R e=U_{\infty} c / v=100 \pm 5$. During the experiments, the flow field is captured with stereo digital particle image velocimetry (DPIV).

Shortly after the initial transient, the flow achieves a steady state at this low Reynolds number. Due to the spatial setup of the oil tank, the maximum travel distance of the plate is limited to 13 chord lengths to avoid the interference of wall effects. In figure 2 , snapshots of the spanwise vorticity $\left(\omega_{z}\right)$ contour are shown for the simulation (with a grid size of $150 \times 66 \times 96$ ) and experiment for the plate 

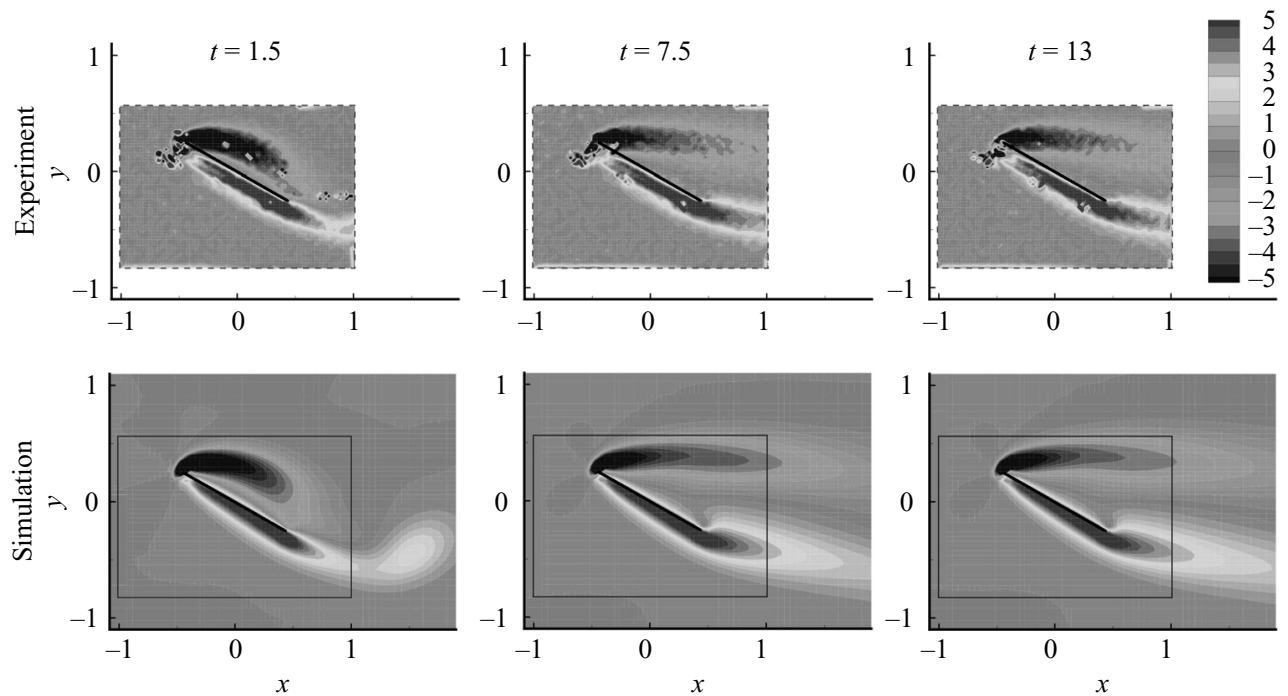

FiguRE 2. Snapshots of spanwise vorticity field at mid-span around a plate of $A R=2$ at $\alpha=30^{\circ}$ from the experiment and the simulation. Free stream is directed from left to right and the position of the plate is illustrated with the black solid line (for the experiment, the position is approximate due to the deflection of the plate). The boxed regions in the simulated results represent the fields of view for the DPIV measurements.

at $\alpha=30^{\circ}$. Following the impulsive start, the leading- and tailing-edge vortices are formed $(t=1.5)$. Later in time, the trailing-edge vortex (starting vortex) detaches and the leading-edge vortex moves upward. At this low Reynolds number, the vorticity profile is very diffusive and the vorticity generated at the leading and tailing edges forms a smeared steady distribution. This flow profile is reached by $t=7.5$ and is indistinguishable from the last snapshot taken from the experiment at $t=13$. As it can be seen from figure 2, the simulated flow field is in good agreement with the DPIV measurements. Three-dimensional flow past a plate of $A R=2$ is found to reach a stable steady state due to the stabilizing effects from the tip vortices and viscosity. We note in passing that the analogous two-dimensional flow at $R e=100$ exhibits periodic shedding of the leading- and trailing-edge vortices.

For the purpose of validating our simulation, we also compare lift and drag forces at $t=13$ from both the simulation and experimental measurements. It is found from the simulation that, past $t=13$, forces decay to the steady value in a very slow manner. Figure 3 compares measured lift and drag coefficients with the simulations based on three different grid sizes for $\alpha \in\left[0^{\circ}, 90^{\circ}\right]$. It can be observed that the two cases with finer grid sizes of $150 \times 66 \times 96$ and $200 \times 88 \times 128$ are almost indistinguishable. Similar grid resolution studies are performed for the cases investigated in the present paper.

Lift coefficients from experiments and simulations are in agreement over the full range of $\alpha$. Drag coefficients show slight discrepancy between the experiment and the numerical computation, due to the thickness of the flat plate. In experiments, the thickness of the plate is $0.037 c$, while in the simulations, we attempt to model an infinitely thin plate with regularized body forces dependent on the grid spacing. However, the flow features are insensitive to our selection of grid spacing at the 

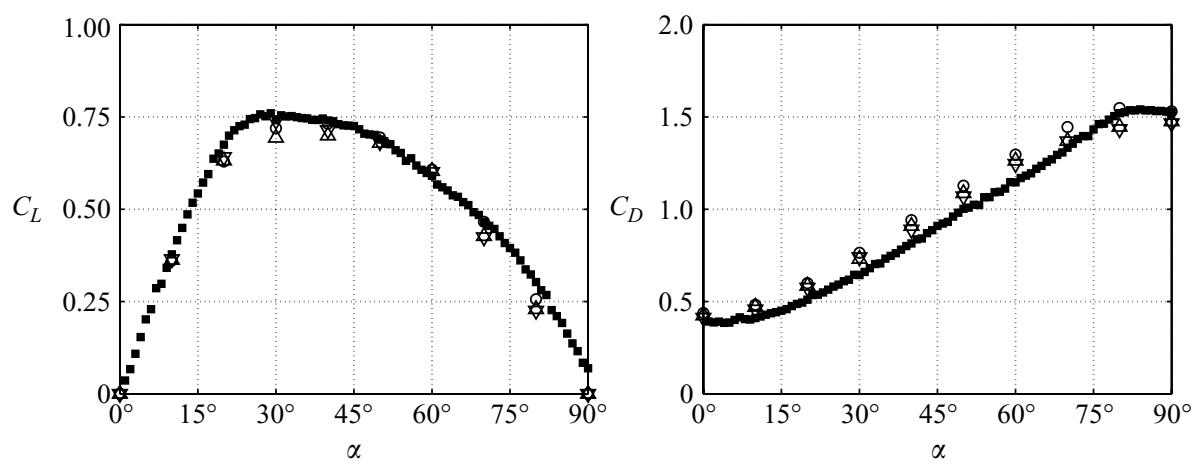

FIGURE 3. Lift and drag coefficients for a rectangular plate of $A R=2$ at $R e=100$. Data from the oil tow tank experiments are shown with (ם) and results from numerical simulations are presented with $(\bigcirc),(\triangle)$ and $(\nabla)$ for grid sizes of $125 \times 55 \times 80,150 \times 66 \times 96$ and $200 \times 88 \times 128$, respectively.

current Reynolds numbers of interest. The shape of the leading or trailing edge (sharp or rounded) has been found to result in no important change in the lift and drag coefficients aside from some difference in the momentum coefficient at higher Reynolds numbers of $\mathcal{O}\left(10^{4}-10^{5}\right)$ (Pelletier \& Mueller 2000; Torres \& Mueller 2004). We also note that the measured forces on the plate may be influenced by the presence of the sting on one of the wing tips.

\section{Separated flows around low-aspect-ratio flat plates}

\subsection{Dynamics of wake vortices behind rectangular planforms}

We first consider the formation and evolution of wake vortices behind rectangular flat plates of $A R=1,2$ and 4 at $R e=300$. Snapshots in figure 4 show the corresponding flow fields at a high angle of attack of $\alpha=30^{\circ}$. The wake vortices are visualized with two different iso-surfaces. First, constant vorticity surface $\left(\|\boldsymbol{\omega}\|_{2}=3\right)$ is shown in light grey to capture the vortex sheets. Second, the second invariant of the velocity gradient tensor $(\nabla \mathbf{u})$, known as the $Q$-criterion or $Q$-value, is used to highlight the vortex cores depicted by the dark grey surfaces $(Q=3)$. Positive $Q$-values give prominence to regions of high swirl in comparison to shear to represent coherent vortices (Hunt, Wray \& Moin 1988). In incompressible flows, the $Q$-value is provided by $Q \equiv \frac{1}{2}\left(\|\boldsymbol{\Omega}\|^{2}-\|\boldsymbol{S}\|^{2}\right)$, where $\boldsymbol{\Omega} \equiv \frac{1}{2}\left[\nabla \boldsymbol{u}-(\nabla \boldsymbol{u})^{T}\right]$ and $\boldsymbol{S} \equiv \frac{1}{2}\left[\nabla \boldsymbol{u}+(\nabla \boldsymbol{u})^{T}\right]$.

Plates are impulsively translated in an initially quiescent flow, generating strong vortex sheets on the top and bottom surfaces of the plates at $t=0^{+}$. Thereafter, the flow separates from the leading edge and tips, which act as strong sources of vorticity. Vorticity is then fed into the flow as vortex sheets that roll up into the leading-edge, trailing-edge and tip vortices. As the leading-edge vortex forms and the trailing-edge vortex advects downstream, the plate experiences a large increase in lift. This enhanced lift is generated by the low-pressure core of the initial leading-edge vortex and is an added effect on top of the lift achieved at large time (also observed in two-dimensional flows by Dickinson \& Götz 1993). Note that the initial topology of the wake structures are the same for all finite $A R$ considered here and the phenomena resemble dynamic stall observed behind high-aspect-ratio wings undergoing a swift pitch up (Carr 1988). 

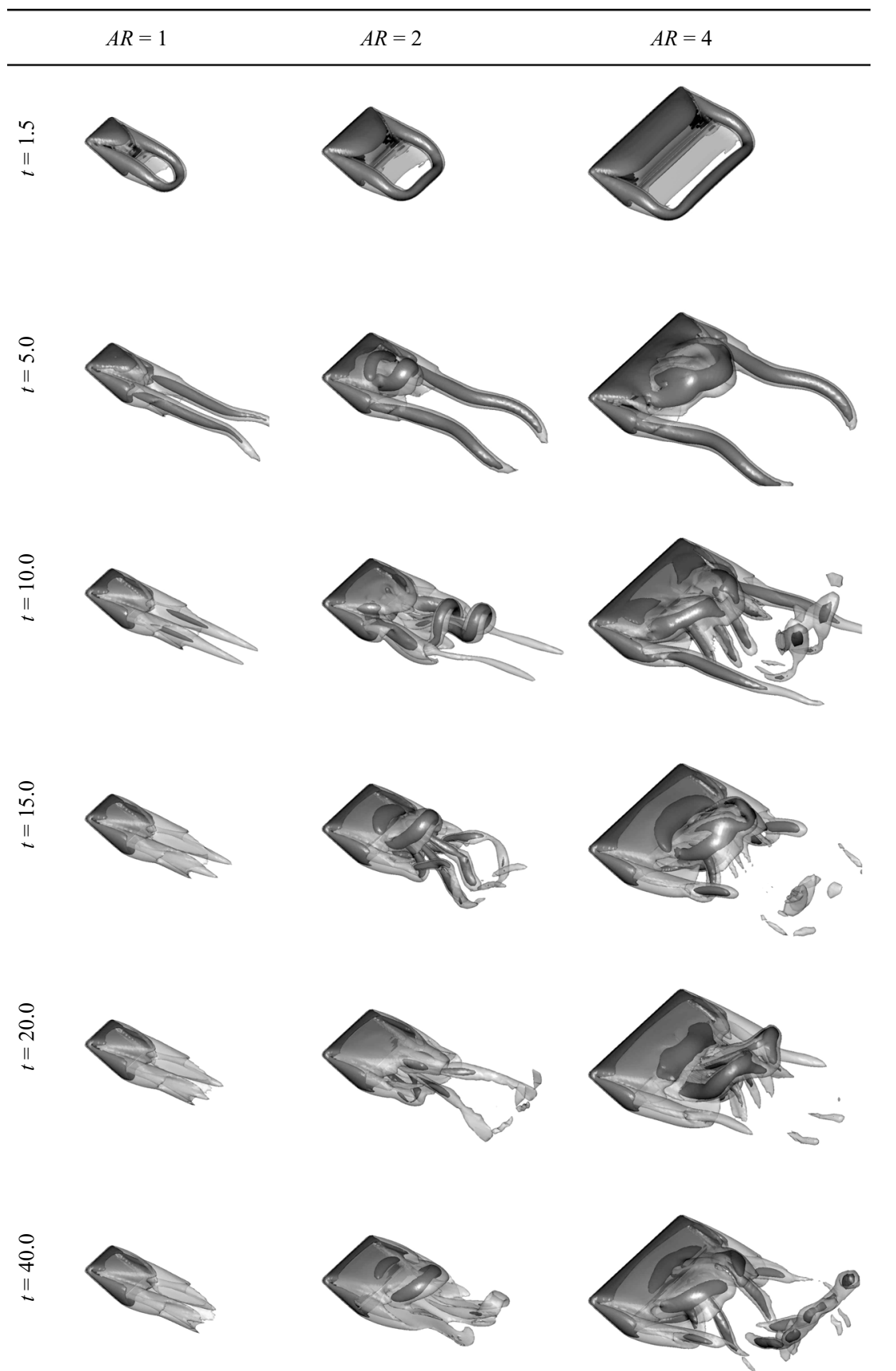

FIGURE 4. Top-port views of the wake vortices behind rectangular plates of $A R=1,2$ and 4 at $\alpha=30^{\circ}$ and $R e=300$. Shown are the iso-surface of $\|\omega\|_{2}=3$ in light grey with vortex cores highlighted by the iso-surface of $Q=3$ in dark grey. 
As features from the initial transient lose their effect on the plate through advection and diffusion, the wake behind the plate becomes strongly dependent on the aspect ratio. At $\alpha=30^{\circ}$, the $A R=1$ case slowly reaches a steady state with a pair of strong counter-rotating tip vortices that cover the entire span of the plate. The vortex sheet created from the leading edge is kept attached to the plate due to the downward induced velocity from the tip vortices.

For a plate of $A R=2$, the vortex sheet emanating from the leading edge rolls into a leading-edge vortex that accumulates spanwise vorticity over time. The tip vortices are not strong enough to keep the leading-edge vortex attached. Around $t \approx 8$, two consecutive leading-edge vortices start to pinch off from the plate. As the detachment takes place, the disconnected vortices start to interact with the tip vortices. This interaction results in the loss of the columnar structures initially maintained by the tip vortices and reduces the downward induced velocity onto the vortical structure residing above the top surface of the plate. Hence, once the initial leading-edge vortices are separated, consecutive formation of the leading-edge vortices (hairpin vortices) by the roll-up of the leading-edge vortex sheet occurs farther and at a higher position. Additionally, weakening of the tip vortices allows the trailing-edge vortex sheet to morph into hairpin vortices that shed. The nonlinear interaction of the wake vortices results in an unsteady aperiodic flow at large time. While it is not apparent from figure 4, there is slight asymmetry in the spanwise direction that contributes to the aperiodic nature of the shedding. Discussion on this asymmetry is offered later in $\S 3.4$.

For the largest finite aspect-ratio plate considered $(A R=4)$, the weaker influence of the tip vortices across the span results in more strongly pronounced periodic shedding of the leading- and trailing-edge vortices. The shedding frequency (nondimensionalized as the Strouhal number with the frontal projection of the chord) for $A R=4$ is found to be $S t \equiv f c \sin \alpha / U_{\infty}=0.12$. In contrast, the two-dimensional shedding frequency for the same Reynolds number and angle of attack is $S t=0.16$.

Around $A R=3$, the vortical structures from the leading and trailing edges start to separate into two cells across the span. The cellular pattern referred to as stall cells becomes more apparent for $A R=4$ where a pair of hairpin vortices are generated from the leading edge and another pair is created from the trailing edge resulting in a release of four hairpin vortices per shedding cycle. Such flow features were also reported on the top surface of the airfoils with oil film and tuft visualizations by Winkelmann \& Barlow (1980) and Yon \& Katz (1998), respectively, at $R e=\mathcal{O}\left(10^{5}\right)$. While we do not notice features of the stall cells directly on the top surface, we find qualitative agreement for the number of cells observed some short distance into the wake.

Despite the interactions between the leading-edge and tip vortices, these vortices remain distinct without merging for all three-dimensional cases. Due to the existence of the right-angled corners on the rectangular plates, the vortex sheets thin out near these regions and the sheets roll up into individual core structures of leadingedge and tip vortices. The separation of the vortical structures indicates a lack of convective vorticity flux in the spanwise direction (i.e. from the mid-span to the tips). Such transport has been suggested to stabilize the leading-edge vortex for flapping wings (Birch \& Dickinson 2001; Birch et al. 2004). For the translating rectangular wings, there is no mechanism to relieve the vorticity being fed into the leading-edge vortex other than diffusion and shedding of the vortical structures. The effects of removing sharp corners by using different planform geometries are discussed later in $\S 3.5$. 

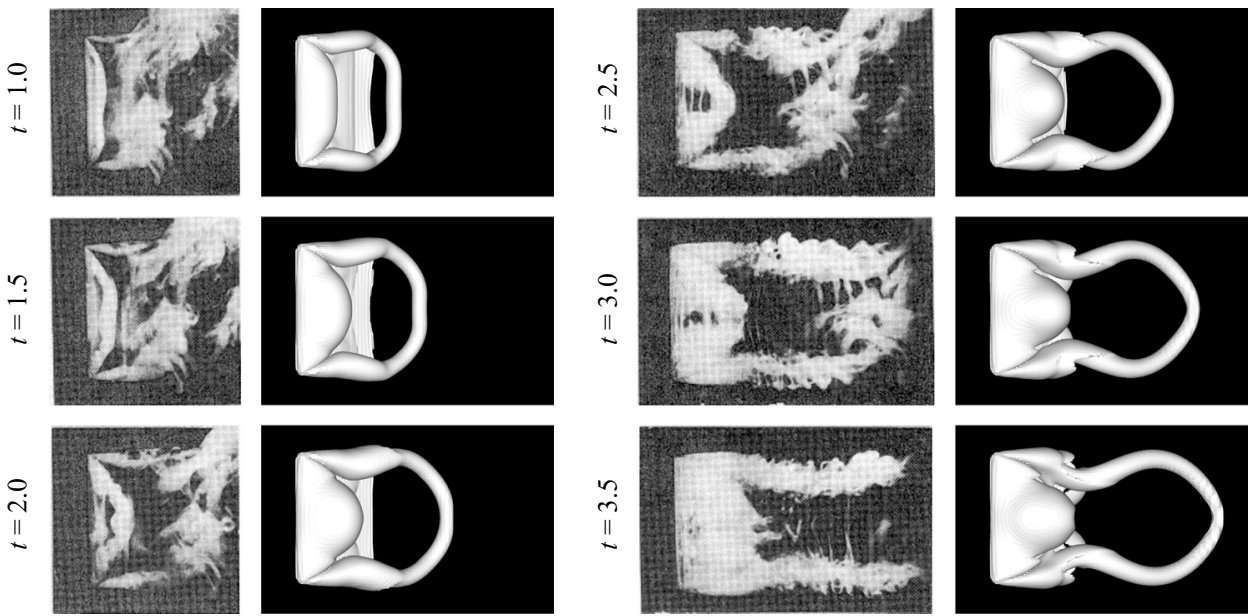

FIgURE 5. Top views of the wake vortices behind a rectangular wing of $A R=2$ at $\alpha=40^{\circ}$ from smoke visualizations at $R e_{a}=5200$ and present results at $R e=500$ with iso-contour of $\|\boldsymbol{\omega}\|_{2}=5$. Smoke visualizations are from Freymuth et al. (1987); reprinted by permission of the American Institute of Aeronautics and Astronautics.

\subsection{Flows at higher Reynolds number}

Flows behind rectangular plates at $R e=500$ are also simulated and are found to be similar to the ones presented here for $R e=300$. With the larger Reynolds number, the wake vortices are less diffused but the topology of the vortical structure are qualitatively similar, which was also noted by Dong et al. (2006) for flows around flapping foils for $R e=100-1000$.

The geometries of the wake vortices in low-Reynolds-number flow $(R e=\mathcal{O}(100))$ at early times following the impulsive start also resemble those in flows of much higher Reynolds numbers, due to the fact that the viscous time scale $\left(t_{v i s} \sim c^{2} / \nu\right)$ is much larger than the time scale associated with advection or acceleration $\left(t_{a d v} \sim c / U_{\infty}\right.$ or $t_{a c c} \sim c^{1 / 2} / a^{1 / 2}$, respectively).

Impulsive flow over a plate of $A R=2$ at $R e=500$ and $\alpha=40^{\circ}$ is simulated and is compared to the smoke visualizations of vortices under a constant acceleration from quiescent flow in a starting wind tunnel (Freymuth et al. 1987) as shown in figure 5. Reynolds number for this experiment is defined with the constant acceleration, the chord and the kinematic viscosity as $R e_{a}=a^{1 / 2} c^{3 / 2} / v=5200$ following the nondimensionalization by Freymuth et al.

As acceleration of immersed boundaries contributes to the generation of vorticity (Hornung 1989), the formation of vortices behind a plate under impulsive translation and constant acceleration cannot be directly compared. However, the formation of start-up vortices should be qualitatively similar at early times before viscous effect significantly influences the flow and the induced velocity of each wake vortex becomes large. The formation of the start-up vortices is illustrated by the snapshots in figure 5 with smoke visualization and the vorticity norm iso-surface.

For the case of constant acceleration, a characteristic velocity of $u_{a}=a^{1 / 2} c^{1 / 2}$ is used to non-dimensionalize the temporal variable. Accordingly, the flow fields are compared at the non-dimensional times of $t U_{\infty} / c$ and $t a^{1 / 2} / c^{1 / 2}$ for the simulations and the experiments, respectively. In figure 5 , it can be seen that the formation and evolution of leading-edge and tip vortices are in good agreement between the 
experiment and the numerical solution despite the difference in the Reynolds number and the velocity profile to which the plate is subjected. The exact location of the trailing-edge vortex from the smoke visualization is difficult to pinpoint but is found to be in accord by its faint trail of smoke at earlier times. The simulation is able to capture even the thin layer of vortex sheet emanating from the leading edge, which would correspond to the region directly downstream of the leading edge that is not visualized by the smoke. Dominant flow features at early times in highReynolds-number flows are captured even with the present low-Reynolds-number flow simulations.

\subsection{Force exerted on the plate}

Unsteady forces on accelerating airfoils at low Reynolds numbers have been considered for two-dimensional flows by Dickinson \& Götz (1993) and Pullin \& Wang (2004). In this section, we consider the forces exerted upon the plate with the three-dimensional wake vortices both immediately after the impulsive start and also at large times.

Representative lift and drag on rectangular plates from the present simulations are presented in figure 6 for $R e=300$. Here results for angles of attack of $\alpha \in\left[0^{\circ}, 60^{\circ}\right]$ and aspect ratios of 2 and 4 as well as the two-dimensional cases are shown for $t \in[0,70]$. At $t=0^{+}$, the impulsive start imposes infinite acceleration on the airfoil in the streamwise direction and results in infinite initial drag (not shown for graphical clarity). Subsequently, lift starts to increase as accumulation of spanwise vorticity instigates the formation of the leading-edge vortex. This increase in lift continues to about $t \approx 1.7$ to reach its maximum. The time to reach maximum lift is observed to be fairly constant in the case of finite aspect ratio wings over most of the angles of attack considered here at low Reynolds numbers. The universality of this number is discussed in detail later on.

After the initial start-up, lift is reduced by as much as half of the maximum value at large time, as shown in figure 6. Depending on whether the wake at large time becomes steady or unsteady, the corresponding force coefficients reach constant or fluctuating values.

It should be noted that the three-dimensional flows under consideration are vastly different from the two-dimensional case, where one observes periodic shedding of the leading- and trailing-edge vortices creating the von Kármán vortex street. Due to the absence of the tip vortices, the two-dimensional flow exerts a strikingly larger fluctuation in force per unit span as shown in figure 6 .

The effect of aspect ratio on the forces is considered by comparing the maximum lift during the transient and the time-averaged forces at large time. These values for wings of $A R=1,2$ and 4, as well as the two-dimensional case, are presented in figure 7 accompanied by their inviscid limits. Stronger influence of downwash from the tip vortices results in reduced lift for lower aspect-ratio plates. For the limiting case of two-dimensional flow, the maximum lift is much higher due to the absence of tip effects (figure 7a). It is interesting to note that the maximum lift achieved soon after the impulsive start is comparable or higher than the three-dimensional inviscid limit for low-aspect-ratio straight wings in incompressible flow (Helmbold 1942):

$$
C_{L}=\frac{2 \pi \alpha}{\sqrt{1+(2 / A R)^{2}}+2 / A R} .
$$

This limit is derived from the lifting surface theory for elliptic wings and is shown to be in remarkable agreement with wings of $A R<4$. Lift for rectangular wings of 

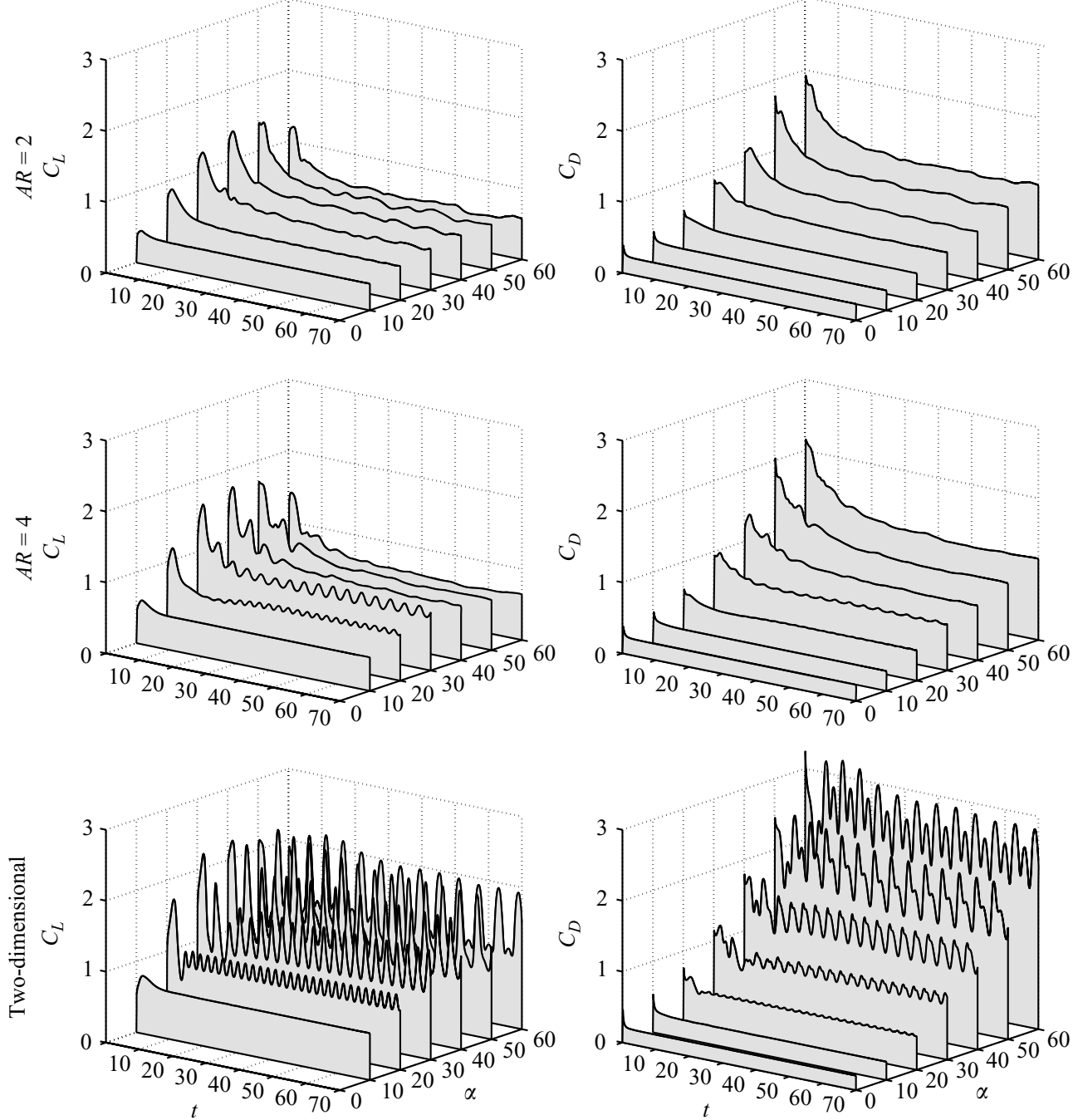

FiguRE 6. Force history on rectangular flat plates of different $A R$ for a range of angles of attack at $R e=300$. Lift and drag coefficients are shown on the left and right panels, respectively.

$0.5 \leqslant A R \leqslant 6.0$ is accurately predicted with this equation as shown in Anderson (1999).

We observe agreement between the time-averaged low-Reynolds-number lift at large time and the above inviscid model at low angles of attack $\left(\alpha \lesssim 10^{\circ}\right)$, where the flows are still attached (figure $7 b$ ). The difference at low angles of attack can be attributed to viscous effects. However, once the flow separates from the plate at higher $\alpha$, the inviscid approximation is no longer able to model the lift behaviour. The high value of $\alpha_{C_{L} \max }$ (angle of maximum lift) was also reported for low-aspect-ratio wings at $R e \cong 10^{5}$ by Torres \& Mueller (2004). We mention that the difference between the maximum $\left(\max C_{L}\right)$ and the average $\left(\operatorname{avg} C_{L}\right)$ lifts is the lift enhancement generated by the initial leading-edge vortex. 

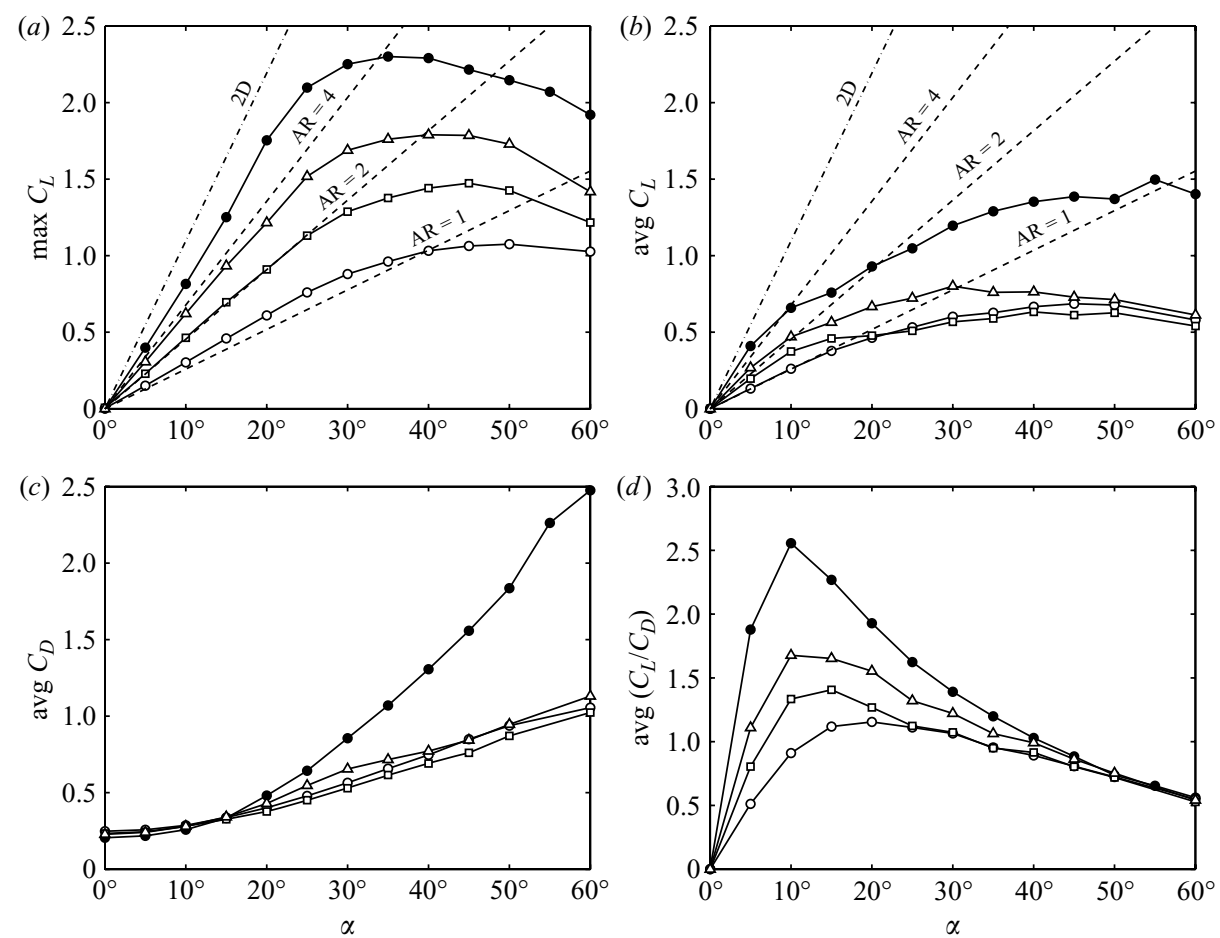

FIGURE 7. Characteristic coefficients for rectangular plates at $R e=300:(a)$ maximum lift; $(b)$ time-averaged lift coefficient at large time; $(c)$ time-averaged drag coefficient at large time and (d) time-averaged lift-to-drag ratio at large time for $A R=1(\bigcirc), A R=2(\square), A R=4(\triangle)$ and two-dimensional $(\bullet)$. Overlaid are the two- $\left(--{ }_{-}\right)$and three-dimensional $(----)$inviscid lift limits.

The average drag values at large time $\left(\operatorname{avg} C_{D}\right)$ for pre-stall angles of attack increase with decreasing aspect ratio (figure $7 c$ ). However for higher angles of attack, drag coefficients are significantly smaller for finite-aspect-ratio wings in contrast to their two-dimensional analogue. It is interesting to note that both average lift and drag coefficients become larger past $\alpha=20^{\circ}$ for $A R=2$ wings than those of $A R=1$, most likely due to the difference in the behaviour of the wake at large time (discussed later in $\S 3.4)$.

At $R e=300$, the viscous stress has a significant influence on the drag experienced by the wing, especially at low angles of attack. In comparison, at $R e=10^{5}$ and $A R=1$, Torres \& Mueller (2004) report $C_{D}=0.025$ and 0.11 for $\alpha=0^{\circ}$ and $10^{\circ}$, respectively. At $\alpha=15^{\circ}, C_{D}=0.24$ is recorded by Torres and Mueller, a value close to what is measured in the current study also (figure $7 c$ ). Hence, we argue that past this angle of attack, pressure drag is the main cause of drag.

Shown also in figure $7(d)$ are the average lift-to-drag ratios at large time $\operatorname{avg}\left(C_{L} / C_{D}\right)$, which are larger for higher aspect-ratio wings. The ratios peak at higher angles of attack for low aspect-ratio plates. While the two-dimensional $\operatorname{avg}\left(C_{L} / C_{D}\right)$ reaches its maximum around $\alpha=10^{\circ}$, that of $A R=1$ is achieved near $\alpha=20^{\circ}$. This may suggest favourable operating conditions at higher angles of attack for the lowaspect-ratio wings. For high angles of attack $\left(\alpha>40^{\circ}\right)$, the lift-to-drag ratio for different aspect ratios coalesce to the same value. 
(a)

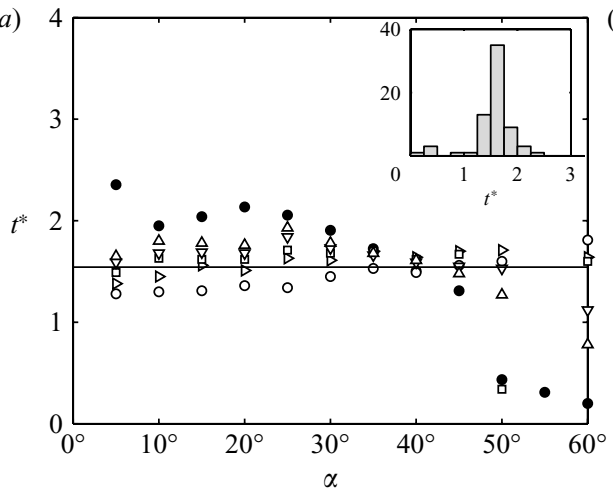

(b)

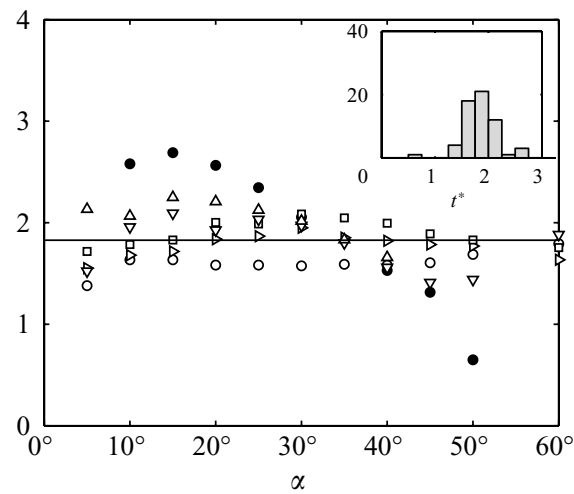

FiguRE 8. Time at which lift achieves the maximum, $t^{*}$, after an impulsive start for different angles of attack at $(a) R e=300$ and $(b) R e=500$. Symbols denote cases for $A R=1(\bigcirc)$, $A R=1.5(\triangleright), A R=2(\square), A R=3(\nabla), A R=4(\triangle)$ and two-dimensional $(\bullet)$. The mean averages are shown with solid lines. The inset figures are the corresponding histograms.

While it is not shown in this section, forces measured at $R e=500$ are found to be quantitatively and qualitatively similar to those at $R e=300$. Interesting differences between the two Reynolds numbers are observed in the stability of the wake, which is described later in $\S 3.4$.

Next, we call attention to the time at which the maximum lift is achieved. We denote this time by $t^{*}$ and present its value in figure 8 for $R e=300$ and 500. It is found that, for the considered aspect ratios and angles of attack, $t^{*}$ is fairly constant around 1.7 (a value between 1.25 and 2.25) because the profiles of the leading-edge vortices are similar among all cases. As the accumulation of spanwise vorticity generated by the leading edge contributes to the growth of the leading-edge vortex, there is reminiscence to the formation number used to describe the time at which vortex rings can no longer grow larger in strength (Gharib, Rambod \& Shariff 1998). Since the formation number is found to be a universal quantity for a variety of flows (e.g. Jeon \& Gharib 2004; Milano \& Gharib 2005), it is not surprising that $t^{*}$ is also fairly constant for the three-dimensional cases considered here. In the case of the two-dimensional flow, we observe a wider range of $t^{*}$ between 1.3 and 2.4 for $\alpha<45^{\circ}$. At higher $\alpha$, a second local maximum starts to emerge for the two-dimensional flow lowering $t^{*}$ significantly.

The side force $\left(F_{z}\right)$ remained zero for all cases that reached steady or periodic unsteady flows. However, for aperiodic flow cases observed at high angles of attack, the wake became asymmetric about the mid-span and exerted side forces upon the plate. These unsteady side forces were an order of magnitude smaller than the dominant lift and drag forces experienced by the plate. For all cases considered in this paper (later summarized in figure 10), it was observed that side forces have small magnitudes of $\left|C_{S}\right|=\left|F_{z} /\left(\frac{1}{2} \rho U_{\infty}^{2} A\right)\right|<0.02$. The genesis of these side forces is discussed in the next section.

\subsection{Large-time behaviour and stability of the wake}

Here, we consider the behaviour of the wake behind rectangular plates at large time. After the initial transient generated by the impulsive start settles down, the wake reaches one of the three states: (i) a stable steady state, (ii) a periodic unsteady state or (iii) an aperiodic unsteady state. Examples from each one of these states were presented in $\S 3.1$. In this subsection, we consider a wide range 


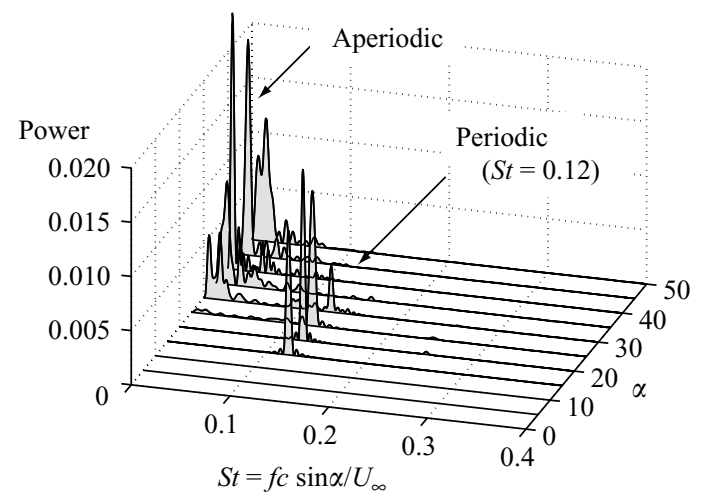

FIGURE 9. Power spectra of the lift trace for a rectangular plate of $A R=3$ in flows of $R e=500$ at various angles of attack.

(a)

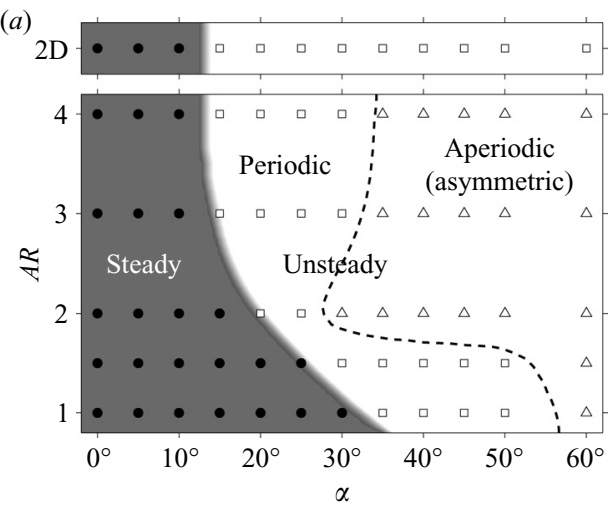

(b)

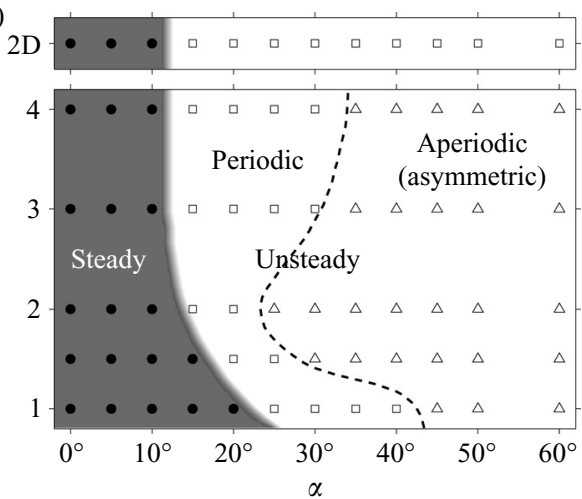

FIGURE 10. Stability of the wake for a range of $\alpha$ and $A R$ at $(a) R e=300$ and $(b) R e=500$. Symbols $(\bullet),(\square)$ and $(\triangle)$ denote steady, unsteady periodic and unsteady aperiodic wakes at large time. The shaded area and the dashed line approximately represent the region of stability and the transition from periodic to aperiodic shedding, respectively. Shown at the top are the two-dimensional findings.

of parameters $\left(\alpha \in\left[0^{\circ}, 60^{\circ}\right], A R=\{1,1.5,2,3,4\}\right.$ and $\left.R e=\{300,500\}\right)$ as well as the two-dimensional flows to survey the stability of the wake at large time. The lift histories, such as the ones in figure 6, are analysed with Fourier transform to detect any dominant shedding frequencies as shown in figure 9 for an example of $R e=500$ and $A R=3$. Depending on the angle of attack, the shedding can occur with a dominant shedding frequency (periodic) or with no clearly recognizable frequency (aperiodic). The dominant frequency in the case of figure 9 is found to be $S t=f c \sin \alpha / U_{\infty}=0.12$ for all spectra of the periodic shedding cases. The corresponding Strouhal number for the two-dimensional flow at $R e=500$ is slightly higher at $S t=0.14-0.16$.

The wake stability is summarized in figure 10 , which maps $\alpha$ against $A R$. These two parameters were found to be the two most important parameters in determining the stability of the wake at large time. Suggested boundaries between different flow regimes are drawn based on the data points collected from numerical experiments. The shaded regions correspond to flow conditions that would arrive at a steady state. Such flow can be either attached at small $\alpha$ or fully separated at moderately high $\alpha$. The steady state is achieved over a wider limit with lower aspect ratios since the tip 
vortices are able to provide a downward induced velocity across a larger extent to prevent the wake vortices from shedding.

As we consider higher angles of attack, the flow exhibits periodically shedding hairpin vortices generated by the leading and/or the trailing edges. This flow profile is observed for the white region at the left of the dashed line in figure 10. The change in the dynamics between the shaded (sub-critical) and unshaded (super-critical) regions can be viewed as an extension of the two-dimensional stability boundary. We claim that the change in the dynamics is attributed to a Hopf-bifurcation, as shown by Ahuja et al. (2007) for the two-dimensional case. For lower aspect ratios, the vortex sheet emanating from the trailing edge forms and sheds hairpin vortices repeatedly. The narrow region with $A R \lesssim 2$ in figure 10 corresponds to such flow states. The same region with higher aspect ratio of $A R \gtrsim 2$ shows shedding of both the leadingand tailing-edge vortices alternately in a periodic fashion (for instance the case of $R e=300, A R=4$ and $\alpha=30^{\circ}$ in figure 4 ).

With further increase in the angle of attack, the tip vortices become more vertically aligned. Essentially, the wake now is comprised of four vortices of similar strengths, namely the leading- and trailing-edge vortices as well as a pair of tip vortices. The tip vortices strongly interact with the leading- and trailing-edge vortices and suppresses the dominant shedding frequency. The transition from periodic to aperiodic flow is illustrated in figure 9 as the peak for the power spectrum at $S t=0.12$ becomes no longer observable for $\alpha>30^{\circ}$. In figure 10, this aperiodic unsteady state corresponds to the region on the right side of the dashed line.

The aperiodic flows are found to be asymmetric in the spanwise direction with respect to the mid-span plane. As the wake becomes asymmetric, the wake vortices apply side forces onto the wing and the flow field. The combination of the asymmetry and the nonlinear interaction among the leading-edge, trailing-edge and tip vortices give rise to the aperiodic nature of the flow. An example of an asymmetric wake is shown in figure 11 for a rectangular plate of $A R=2$ at $\alpha=40^{\circ}$ and $R e=500$ (the wake for the same case at earlier time is shown in $\S 3.2$ ). The side force for this case has a magnitude of $\left|C_{S}\right|<0.01$ with a frequency content (no dominant shedding frequency) similar to those low-frequency contents shown in figure 9. Asymmetry is not observed for steady or periodic unsteady flows.

For much larger aspect ratios than those considered here, the wake most likely develops into either a stable steady state or a periodic shedding profile. However, the actual three-dimensional flow with infinite span would probably not be purely two-dimensional, as seen for three-dimensional flows around a circular cylinder of infinite span (Braza, Faghani \& Persillon 2001). Spanwise perturbations can induce the creation of spanwise vorticity and the corresponding spanwise undulations. Hence, formation of cellular vortical patterns (stall cells) can be observed directly above the top surface, similar to the structures seen in the case with $A R=4$ (figure 4) and those previously reported by Winkelmann \& Barlow (1980) and Yon \& Katz (1998).

The stability of the wake is also influenced by the Reynolds number. In figure 10, we notice that for $R e=500$, the steady flow profile is achieved for a smaller range of angles of attack and aspect ratios compared to the case where $R e=300$. Similar trend holds for the periodic shedding case. With increasing Reynolds number, it is expected that the wake to exhibit strong interaction between the leading-edge and tip vortices resulting in aperiodic/asymmetric flows for a wider combination of angles of attack and aspect ratios. At higher Reynolds numbers, it may be possible to observe changes in the shape of the stability boundary between the periodic and aperiodic shedding regimes. 


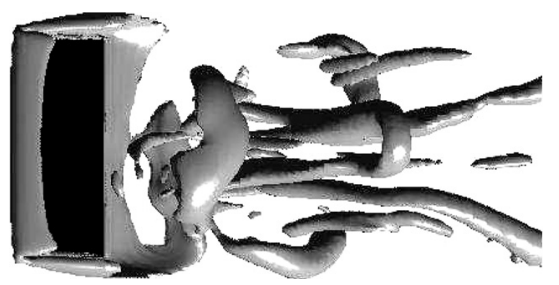

FIGURE 11. Top view of the asymmetric wake at large time behind a rectangular plate of $A R=2$ at $\alpha=40^{\circ}$ and $R e=500$. Vortices are highlighted with iso-contours of $Q=2.5$. The flow is directed from left to right and the wing is shown in black.

\subsection{Non-rectangular planforms}

Stable attachment of the leading-edge vortices on flapping wings has been observed to provide enhanced lift for prolonged duration (Birch et al. 2004; Poelma et al. 2006). Shedding of these vortices are prevented by releasing the spanwise vorticity through convective transport from the root to the tip of the wings induced by wing rotation. Hence, a continuously connected vortical structure formed by the leading-edge and tip vortices are observed for flapping or revolving wings.

For rectangular plates in pure translation, we have shown earlier that the initial leading-edge vortex detaches in a similar fashion to dynamic stall. The leading-edge and tip vortices remain as separate vortical structures and do not provide a mechanism for the spanwise vorticity to be released other than shedding. In order to prevent or delay the shedding of the leading-edge vortex, we consider the use of curved or angled leading edges to induce flows along the leading edge. Flows around elliptic, semicircular and delta-shaped planforms are simulated at $\alpha=30^{\circ}$ and $R e=300$ in comparison to the flow around a rectangular plate of $A R=2$ presented earlier. The geometries of the elliptic, semicircular and delta-shaped planforms are chosen with $A R=2,4 / \pi$ and 4 , respectively, whose mean chord lengths $(c \equiv A / b$, where $b$ is the wing span) are used to non-dimensionalize all spatial variables. For the delta wing, the sweep angle is set to $45^{\circ}$.

Wake structures behind the non-rectangular planforms are shown in figure 12 after the impulsive start with the corresponding forces in figure 13. For the elliptic and semicircular cases, there are no discontinuities in the vortex sheet that emanates from the leading edge to the tips, unlike the sharp separation of the vortical structures around the corners on the rectangular planforms. The curved leading edge encourages spanwise transport of vorticity into the tip vortices. Hence to some extent the shedding of the leading-edge vortical structure is delayed. While the leading-edge hairpin vortex first detaches around $t \approx 8$ for the rectangular planform, such separation is observed at a later time $(t \approx 15)$ for the elliptic and semicircular plates. This gentle detachment allows the forces to decay smoothly instead of generating fluctuations, as seen for the rectangular planform around $t \approx 8$ due to the detachment of two consecutive leading-edge vortices. At later time, both flows behind the elliptic and semicircular wings exhibit periodic shedding, which is different from the rectangular case.

A steady state is achieved by the flow around the delta-shaped planform. There is a clear distinction of the left and right vortex sheets from the nose of the plate, but the roll-up of the vortex sheets forms a stable wake structure which in turn attains steady lift and drag. The absence of wing tips for this planform allows the vortex sheets from the leading edge to roll up and convect downstream in a very stable manner (see figure 12). For $R e=300$, the size of initial leading-edge vortices is larger 


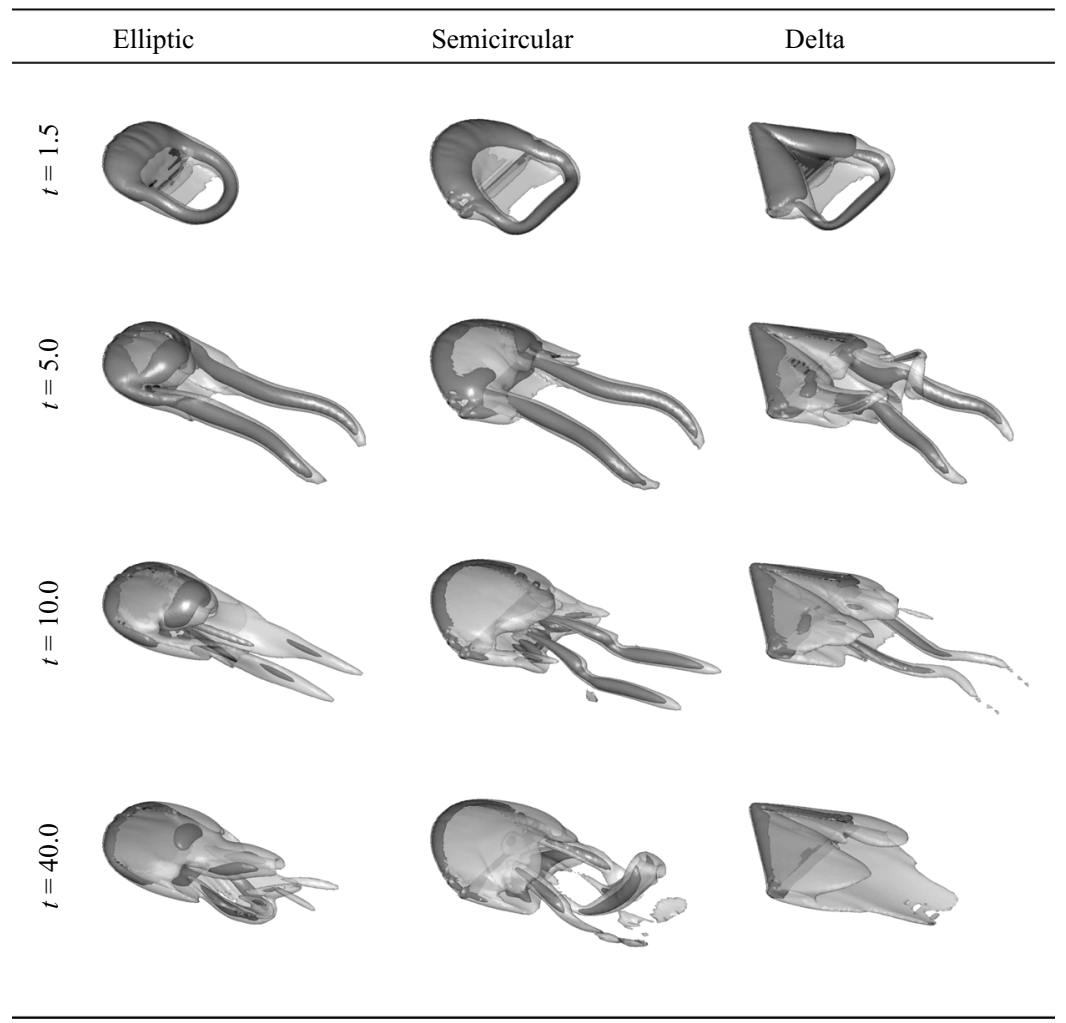

FiguRE 12. Top-port views of the wake vortices behind different planform geometries at $\alpha=30^{\circ}$ and $R e=300$ with the iso-surface of $\|\omega\|_{2}=3$ in light grey with vortex cores highlighted by the iso-surface of $Q=3$ in dark grey.

compared to the ones from higher Reynolds number flow (Gursul, Gordnier \& Visbal 2005). One can observe transient behaviour of the wake until $t \approx 15$. Beyond this point in time, there is some unsteady shedding of small vortical structures behind the rolled up vortices. However, the wake and the forces do not change much past $t \approx 15$ approaching the steady state.

We observe a relative increase in transport of the spanwise vorticity around the non-rectangular planforms in comparison to the rectangular planform as illustrated by iso-surface of $\left|\boldsymbol{u} \cdot \nabla \omega_{z}\right|$ in figure 14. It should be noticed that there is an absence of transport of $\omega_{z}$ near the leading edge for the rectangular wing in contrast to the semicircular and delta-shaped planforms. The force histories presented in figure 13 show that the time of maximum lift is somewhat delayed to $t^{*} \approx 2$ for the elliptic and semicircular wings, in comparison to $t^{*} \approx 1.7$ for the rectangular plate of $A R=2$. Nonetheless, the plates experience a drop in lift due to the separation of the leadingedge vortices later in time. This detachment is caused by the insufficient spanwise transport (release) of spanwise vorticity to sustain a stable attachment of the leadingedge vortex under pure translation.

While the vortical flows are different for various planform geometries, the lift and drag exerted on the wings do not show significant variations in figure 13. This is most likely due to the viscous nature of the flows at this low Reynolds number. In addition, the similar aspect ratios considered here may be responsible for the similar values in forces. We do also observe differences in the bahaviour and stability of the 

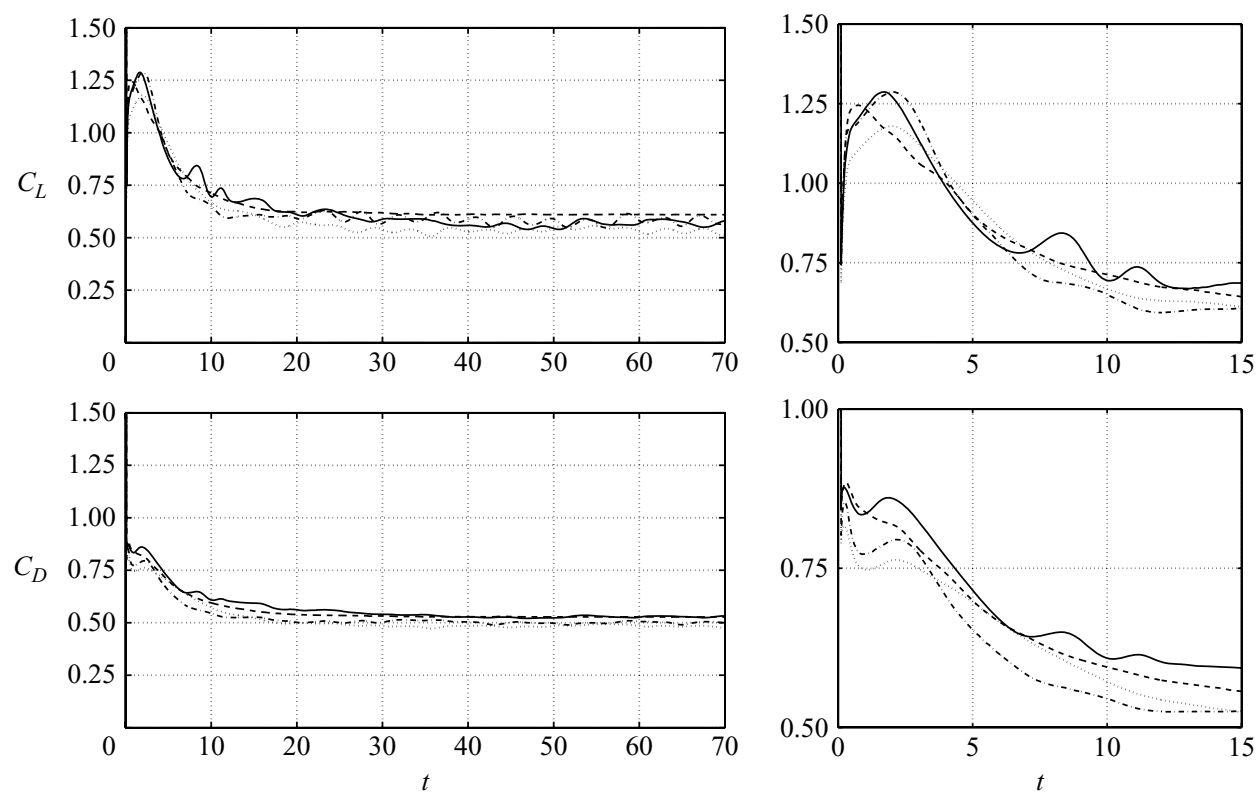

FIGURE 13. Time trace of lift and drag coefficients for rectangular $(-)$, elliptic $(\cdots \cdots \cdots)$, semicircular (-- ) and delta-shaped $(---)^{-}$planforms at $R e=300$ and $\alpha=30^{\circ}$. The figures on the right side magnify the corresponding (early-time) transient behaviour shown in the left-side figures.

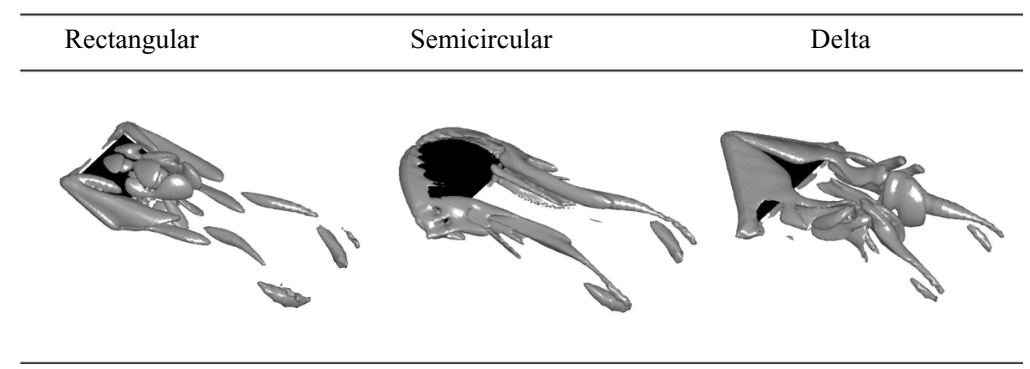

FIGURE 14. Top-port views of the convective transport of spanwise vorticity shown by the iso-surface of $\left|\boldsymbol{u} \cdot \nabla \omega_{z}\right|=3$ at $t=5$.

wakes. The stability diagram presented in figure 10 for the rectangular plate would not carry over to the cases of non-rectangular planforms due the difference in the influence of the tip vortices.

For flapping wing aerodynamics, the wing kinematics restricts the wing stroke and periodicity. In such cases, the travel distance even at the wing tip is much smaller and is about $\pi A R c$. Even a small increase in aerodynamic performance or stable flow features can be of benefit. Although the added effect of rotation or spanwise flows are not taken into account in this study, the wing planform can be of importance as we have discussed in the non-rectangular cases, at least without regard to structural constraints or maneuverability. For purely translating flights, the initially formed leading-edge vortex does not stay attached for most planform geometries as reported around rotating wings. As discussed in previous studies, it is suspected that rotation is one of the main mechanisms for the stable attachment. 


\section{Conclusions}

We presented results from numerical simulations of three-dimensional separated flows around low-aspect-ratio flat-plate wings at low Reynolds numbers using an immersed boundary method. Both the initial transient and long-time behaviour of the flow was studied by simulating an impulsively started plate in pure translation. The unsteady nature of the separated flow and vortex dynamics was highlighted.

A number of simulations were performed for $R e=300$ and 500 with various aspect ratios, angles of attack and planform geometries. The aspect ratio and angle of attack were found to have a large influence on the stability of the wake profile and the force experienced by the body. At early times, topologies of the wake vortices were found to be the same across different aspect ratios and angles of attack. Behind low-aspect-ratio rectangular plates, leading-edge vortices were found to form and eventually separate as hairpin vortices following the initial impulsive translation. This phenomenon was found to be similar to dynamic stall observed behind pitching plates. The detached structure would then interact with the tip vortices, reducing the downward velocity induced by the tip vortices acting upon the leading-edge vortex. At large time, depending on the aspect ratio and angles of attack, the wakes reached one of the three states: (i) a steady state, (ii) a periodic unsteady state or (iii) an aperiodic unsteady state. The aperiodic unsteady state was found to be asymmetric in the spanwise direction and was caused by the strong interaction between the leading-edge, trailing-edge and tip vortices.

Lift achieved the maximum value soon after the impulsive start and decreased afterwards to approximately half of the maximum value at large time. It was observed that for most of the cases maximum lift in time was achieved at a non-dimensional time around $t^{*} \approx 1.7$ regardless of the aspect ratio, angle of attack and planform geometry. We remarked on the reminiscence to the formation number and its possible universality of this non-dimensional time.

Elliptic, semicircular and delta wings were also considered. By providing curvature along the leading edge, convective transport of vorticity from the mid-span to the tip somewhat delayed the separation of the leading-edge vortex. However, the curvature for these planforms could not induce sufficient transport of spanwise vorticity to prevent the shedding of the leading-edge vortices. The wakes behind non-rectangular wings were observed to be different from the cases of rectangular planforms in terms of the shedding pattern (stability) and the influence of the tip vortices. However, the overall trend for the lift and drag histories were found to be similar to those of the rectangular case.

Three-dimensional separated flows behind low-aspect-ratio plates were found to be vastly different from the analogous two-dimensional flows. We have observed that the tip effects in three-dimensional flows can stabilize the flow and also exhibit nonlinear interaction of the shedding vortices. Even when the aspect ratio is increased to 4, the flow along the mid-span does not approach the two-dimensional von Kármán vortex shedding since spanwise cellular structures (stall cells) emerge. Asymmetric wakes about the mid-span were also observed for aperiodic flows around rectangular wings at high angles of attack. Furthermore, the tip vortices and their interaction with the other wake vortices were significantly influenced by the planform geometry.

This work was supported by the US Air Force Office of Scientific Research (FA955005-1-0369) with some of the computations made possible by the US Department of Defense High Performance Computing Modernization Program. We are thankful to Professors C. W. Rowley, M. Gharib, D. R. Williams, G. Tadmor and M. H. Dickinson 
for the enlightening discussions. Experimental data in $\S 2.3$ were generously shared by Dr W. B. Dickson.

\section{REFERENCES}

Ahuja, S., Rowley, C. W., Kevrekidis, I. G., Colonius, T. \& Tadmor, G. 2007 Low-dimensional models for control of leading-edge vortices: equilibria and linearized models. AIAA Paper 2007-709.

Anderson, J. D. 1999 Aircraft Performance and Design. McGraw-Hill.

Birch, J. M. \& Dickinson, M. H. 2001 Spanwise flow and the attachment of the leading-edge vortex on insect wings. Nature 412, 729-733.

Birch, J. M., Dickson, W. B. \& Dickinson, M. H. 2004 Force production and flow structure of the leading edge vortex on flapping wings at high and low Reynolds numbers. J. Exp. Biol. 207, 1063-1072.

Blondeaux, P., Fornarelli, F., Guglielmini, L., Triantafyllou, M. S. \& Verzicco, R. 2005 Numerical experiments on flapping foils mimicking fisk-like locomotion. Phys. Fluids 17, 113601.

Bos, F. M., Lentink, D., Van Oudheusden, B. W. \& Bijl, H. 2008 Influence of wing kinematics on aerodynamic performance in hovering insect flight. J. Fluid Mech. 594, 341-368.

Braza, M., Faghani, D. \& Persillon, H. 2001 Successive stages and role of natural vortex dislocations in three-dimensional wake transition. J. Fluid Mech. 439, 1-41.

Buchrolz, J. H. J. \& SMits, A. J. 2006 On the evolution of the wake structure produced by a low-aspect-ratio pitching panel. J. Fluid Mech. 546, 433-443.

CARr, L. W. 1988 Progress in analysis and prediction of dynamic stall. J. Aircraft 25 (1), 6-17.

Cosyn, P. \& VierendeELS, J. 2006 Numerical investigation of low-aspect-ratio wings at low Reynolds numbers. J. Aircraft 43 (3), 713-722.

Dickinson, M. H. \& GöTz, K. G. 1993 Unsteady aerodynamic performance of model wings at low Reynolds numbers. J. Exp. Biol. 174, 45-64.

Dickson, W. B. \& Dickinson, M. H. 2004 The effect of advance ratio on the aerodynamics of revolving wings. J. Exp. Biol. 207, 4269-4281.

Dong, H., Mittal, R. \& NaJjar, F. M. 2006 Wake topology and hydrodynamic performance of low-aspect-ratio flapping foils. J. Fluid Mech. 566, 309-343.

Drucker, E. G. \& Lauder, G. V. 1999 Locomotor forces on a swimming fish: three dimensional vortex wake dynamics quantified using digital particle image velocimetry. J. Exp. Biol. 202, 2393-2412.

von Ellenrieder, K. D., Parker, K. \& Soria, J. 2003 Flow structures behind a heaving and pitching finite-span wing. J. Fluid Mech. 490, 129-138.

Ellington, C. P., van den Berg, C., Willmott, A. P. \& Thomas, A. L. R. 1996 Leading-edge vortices in insect flight. Nature 384, 626-630.

Freymuth, P., Finaish, F. \& BANK, W. 1987 Further visualization of combined wing tip and starting vortex systems. AIAA J. 25 (9), 1153-1159.

Gharib, M., Rambod, E. \& Shariff, K. 1998 A universal time scale for vortex ring formation. J. Fluid Mech. 360, 121-140.

Gursul, I., Gordnier, R. \& Visbal, M. 2005 Unsteady aerodynamics of nonslender delta wings. Prog. Aero. Sci. 41, 515-557.

Hamdani, H. \& Sun, M. 2000 Aerodynamic forces and flow structures of an airfoil in some unsteady motions at small Reynolds number. Acta Mech. 145, 173-187.

Helmbold, H. B. 1942 Der unverwundene ellipsenflugel als tragende flanche. Jahrbuch 1942 der Deutch Luftfahrtforsch pp. I111-I113.

Hornung, H. 1989 Vorticity generation and transport. 10th Australasian fluid mechanics conference, Paper KS-3.

Hunt, J. C. R., Wray, A. A. \& Moin, P. 1988 Eddies, stream, and convergence zones in turbulent flows. Technical Report CTR-S88. Center for Turbulent Research.

JeOn, D. \& Gharib, M. 2004 On the relationship between the wake vortex formation process and cylinder wake vortex patterns. J. Fluid Mech. 519, 161-181.

Liu, H. \& Kawachi, K. 1998 A numerical study of insect flight. J. Comput. Phys. 146, 124-156. 
Milano, M. \& Gharib, M. 2005 Uncovering the physics of flapping flat plates with artificial evolution. J. Fluid Mech. 534, 403-409.

Mittal, R. \& Iaccarino, G. 2005 Immersed boundary methods. Annu. Rev. Fluid Mech. 37, 239-261.

Mittal, S. \& Tezduyar, T. E. 1995 Parallel finite element simulation of 3D incompressible fluidstructure interactions. Intl J. Numer. Meth. Fluids 21, 933-953.

Parker, K., von Ellenrieder, K. D. \& Soria, J. 2007 Morphology of the forced oscillatory flow past a finite-span wing at low Reynolds number. J. Fluid Mech. 571, 327-357.

Pelletier, A. \& Mueller, T. J. 2000 Low Reynolds number aerodynamics of low-aspect-ratio, thin/flat/cambered-plate wings. J. Aircraft 37 (5), 825-832.

Peskin, C. S. 2002 The immersed boundary method. Acta Numer. 11, 479-517.

Pines, D. J. \& Bohorquez, F. 2006 Challenges facing future micro-air-vehicle development. J. Aircraft 34 (2), 290-305.

Poelma, C., Dickson, W. B. \& Dickinson, M. H. 2006 Time-resolved reconstruction of the full velocity field aournd a dynamically-scaled flapping wing. Exp. Fluids 41, 213-225.

Pullin, D. I. \& WANG, Z. J. 2004 Unsteady forces on an accelerating plate and application to hovering insect flight. J. Fluid Mech. 509, 1-21.

Ringuette, M. J., Milano, M. \& Gharib, M. 2007 Role of the tip vortex in the force generation of low-aspect-ratio normal flat plates. J. Fluid Mech. 581, 453-468.

Sun, M. 2005 High-lift generation and power requirements of insect flight. Fluid Dyn. Res. 37, 21-39.

TAIRA, K. 2008 The immersed boundary projection method and its application to simulation and control of flows around low-aspect-ratio wings. PhD thesis, California Institute of Technology.

TAIRA, K. \& Colonius, T. 2007 The immersed boundary method: a projection approach. J. Comput. Phys. 225, 2118-2137.

Taira, K., Dickson, W. B., Colonius, T., Dickinson, M. H. \& Rowley, C. W. 2007 Unsteadiness in flow over a flat plate at angle-of-attack at low Reynolds numbers. AIAA Paper 2007-710.

TORRES, G. E. \& Mueller, T. J. 2004 Low-aspect-ratio wing aerodynamics at low Reynolds numbers. AIAA J. 42 (5), 865-873.

Usherwood, J. R. \& Ellington, C. P. 2002 The aerodynamics of revolving wings, I model hawkmoth wings. J. Exp. Biol. 205, 1547-1564.

WANG, Z. J. $2000 a$ Two dimensional mechanism for insect hovering. Phys. Rev. Lett. 85 (10), $2216-2219$.

WANG, Z. J. $2000 b$ Vortex shedding and frequency selection in flapping flight. J. Fluid Mech. 410, 323-341.

WANG, Z. J. 2004 The role of drag in insect hovering. J. Exp. Biol. 207, 4147-4155.

Winkelmann, A. E. \& Barlow, J. B. 1980 Flowfield model for a rectangular planform wing beyond stall. AIAA J. 18 (8), 1006-1007.

Yon, S. A. \& Katz, J. 1998 Study of the unsteady flow features on a stalled wing. AIAA J. 36 (3), 305-312.

Zhu, Q., Wolfgang, M. J., Yue, D. K. P. \& Triantafyllou, M. S. 2002 Three-dimensional flow structures and vorticity control in fish-like swimming. J. Fluid Mech. 468, 1-28. 